\title{
Coupled-channel $\Lambda_{c} N-\Sigma_{c} N$ interaction from lattice $Q C D$
}

\section{Takaya Miyamoto*}

Center for Gravitational Physics, Yukawa Institute for Theoretical Physics, Kyoto University, Kyoto 606-8502, Japan

E-mail: takaya.mivamotodyukawa.kyoto-u.ac.jp

\section{for HAL QCD Collaboration}

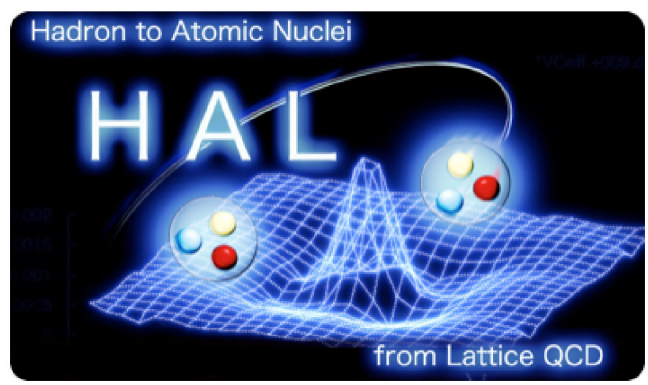

We study S-wave interactions in the $I\left(J^{P}\right)=\frac{1}{2}\left(1^{+}\right) \Lambda_{c} N-\Sigma_{c} N$ system on the basis of the coupled-channel HAL QCD method. The potentials which are faithful to QCD S-matrix below the $\Sigma_{c}^{*} N$ threshold are extracted from Nambu-Bethe-Salpeter wave functions on the lattice. For the simulation, we employ the (2+1)-flavor gauge configurations on a $(2.9 \mathrm{fm})^{3}$ volume at $m_{\pi} \simeq 700,570,410 \mathrm{MeV}$. We present our results of the S-wave coupled-channel potentials for the $\Lambda_{c} N-\Sigma_{c} N$ system in the $\frac{1}{2}\left(1^{+}\right)$state as well as scattering observables obtained from the extracted potential matrix. We observe that the attraction in the $\Sigma_{c} N$ channel is stronger than that in the $\Lambda_{c} N$ channel and the coupling between $\Lambda_{c} N$ and $\Sigma_{c} N$ channels is weak.

XVII International Conference on Hadron Spectroscopy and Structure - Hadron2017

25-29 September, 2017

University of Salamanca, Salamanca, Spain

\footnotetext{
* Speaker.
} 


\section{Introduction}

The study of interactions between a charmed baryon and a nucleon $\left(Y_{c} N\right.$ interactions) is an important subject to investigate the property of the charmed baryon in hadronic matters. It is, however, a challenging problem because we have no experimental data in charmed baryon scatterings as well as charmed hypernuclei so far. Under this circumstance, it is desirable to determine the interactions by the first-principles calculations of QCD, such as lattice QCD simulations.

In the recent years, an approach to investigate hadron interactions in lattice QCD has been proposed by the HAL QCD Collaboration [U, [1]. One of the advantages of the HAL QCD method is that it can be extended straightforwardly to the case of inelastic scatterings. The extended method, namely coupled-channel HAL QCD method [罒], has been applied to the hyperon system [䧃] as well as the charmed meson system [ []]. The scattering amplitude calculated from obtained potentials can be used to compare the scattering observables with experimental data [5].

Employing the HAL QCD method, we have previously investigated the interactions in the $\Lambda_{c} N$ elastic scattering [ [6], as the first step to understand charmed baryon interactions in lattice QCD, and we found that the $\Lambda_{c} N$ interactions are attractive at low energies but not strong enough to form two-body bound states at $m_{\pi} \geq 410 \mathrm{MeV}$. In this report, we extend our consideration to the inelastic regions for the $\Lambda_{c} N$ scattering by using coupled-channel HAL QCD method. In particular, we focus our attention on the S-wave $\Lambda_{c} N-\Sigma_{c} N$ system in $I\left(J^{P}\right)=\frac{1}{2}\left(1^{+}\right)$state. In order to discuss properties of the S-wave $\Lambda_{c} N-\Sigma_{c} N$ interaction, we calculate the scattering observables from obtained potentials in the infinite volume, such as phase shifts for $\Lambda_{c} N$ and $\Sigma_{c} N$ systems as well as the inelasticity of the scatterings.

\section{Coupled-channel HAL QCD method}

We explain the coupled-channel HAL QCD method [B], which will be applied to the $\Lambda_{c} N-\Sigma_{c} N$ system. A key quantity in the HAL QCD method is the equal-time Nambu-Bethe-Salpeter (NBS) wave function which encodes informations of scattering amplitude in its asymptotic behavior [B]]. In the center-of-mass frame, the NBS wave function of two baryons at Euclidean time $t$ with the total energy $W$ is defined by ${ }^{1}$

$$
\psi_{C}^{(W)}(\vec{r}) e^{-W t}=\frac{1}{\sqrt{Z_{C_{1}}} \sqrt{Z_{C_{2}}}} \sum_{\vec{x}}\left\langle 0\left|B_{C_{1}}(\vec{r}+\vec{x}, t) B_{C_{2}}(\vec{x}, t)\right| W\right\rangle,
$$

where the index $C$ denote the flavor channel $\left(\mathrm{C}=\Lambda_{c} N, \Sigma_{c} N\right)$, and $B_{C_{i}}(i=1,2)$ is the local interpolating operator for the baryon $C_{i}$ with its renormalization factor $\sqrt{Z_{C_{i}}}$. In the case of $C=\Lambda_{c} N$, for instance, $C_{1}=\Lambda_{c}$ and $C_{2}=N$. The $|W\rangle$ stands for an QCD asymptotic in-state at the total energy of $W$. From the NBS wave functions, we define the energy independent non-local potentials through the following coupled-channel Schrödinger equation,

$$
\left[E_{C}-\left(H_{0}\right)_{C}\right] \psi_{C}^{(W)}(\vec{r})=\sum_{C^{\prime}} \int d^{3} r^{\prime} U_{C}^{C^{\prime}}\left(\vec{r}, \vec{r}^{\prime}\right) \psi_{C^{\prime}}^{(W)}\left(\vec{r}^{\prime}\right),
$$

\footnotetext{
${ }^{1}$ Here we ignore spin indices in the NBS wave function for simplicity.
} 
where $\left(H_{0}\right)_{C}=-\nabla^{2} / 2 \mu_{C}$ with the reduced mass $\mu_{C}=m_{C_{1}} m_{C_{2}} /\left(m_{C_{1}}+m_{C_{2}}\right)$ and $E_{C}=k_{C}^{2} / 2 \mu_{C}$. The relative momentum $k_{C}$ is determined from the total energy $W=\sqrt{k_{C}^{2}+m_{C_{1}}}+\sqrt{k_{C}^{2}+m_{C_{2}}}$. By definition, the non-local potential $U_{C}^{C^{\prime}}\left(\vec{r}, \vec{r}^{\prime}\right)$ is faithful to the QCD S-matrix unless new channel opens. In order to handle the non-locality of the potentials, we introduce the derivative expansion $U_{C}^{C^{\prime}}\left(\vec{r}, \vec{r}^{\prime}\right)=V_{C}^{C^{\prime}}(\vec{r}, \vec{\nabla}) \delta^{(3)}\left(\vec{r}-\vec{r}^{\prime}\right)$, where $V_{C}^{C^{\prime}}(\vec{r}, \vec{\nabla})$ is then expanded in terms of $\vec{\nabla}$. The leadingorder potential matrix is extracted by using the NBS wave functions as

$$
\left(\begin{array}{cc}
V_{\Lambda_{c} N}^{\Lambda_{c} N}(\vec{r}) & V_{\Lambda_{c} N}^{\Sigma_{c} N}(\vec{r}) \\
V_{\Sigma_{c} N}(\vec{r}) & V_{\Sigma_{c} N}^{\sum_{c} N}(\vec{r})
\end{array}\right)=\left(\begin{array}{cc}
K_{\Lambda_{c} N}^{\left(W_{1}\right)}(\vec{r}) & K_{\Lambda_{c} N}^{\left(W_{2}\right)}(\vec{r}) \\
K_{\Sigma_{c} N}^{\left(W_{1}\right)}(\vec{r}) & K_{\Sigma_{c} N}^{\left(W_{2}\right)}(\vec{r})
\end{array}\right)\left(\begin{array}{cc}
\psi_{\Lambda_{c} N}^{\left(W_{1}\right)}(\vec{r}) & \psi_{\Lambda_{c} N}^{\left(W_{2}\right)}(\vec{r}) \\
\psi_{\Sigma_{c} N}^{\left(W_{1}\right)}(\vec{r}) & \psi_{\Sigma_{c} N}^{\left(W_{2}\right)}(\vec{r})
\end{array}\right)^{-1}
$$

where $K_{C}^{(W)}(\vec{r}) \equiv\left[E_{C}-\left(H_{0}\right)_{C}\right] \psi_{C}^{(W)}(\vec{r})$.

In lattice QCD, the NBS wave functions can be extracted from the baryon four-point correlation function given by

$$
\begin{aligned}
G_{C}^{\tilde{C}}\left(\vec{r}, t-t_{0}\right) & =\sum_{\vec{x}}\left\langle 0\left|B_{C_{1}}(\vec{r}+\vec{x}, t) B_{C_{2}}(\vec{x}, t) \overline{\mathscr{J} \tilde{C}}\left(t_{0}\right)\right| 0\right\rangle \\
& =\sum_{n} \sqrt{Z_{C_{1}}} \sqrt{Z_{C_{2}}} \psi_{C}^{\left(W_{n}\right)}(\vec{r}) e^{-W_{n}\left(t-t_{0}\right)} A_{n}^{\tilde{C}}+\cdots,
\end{aligned}
$$

with constant $A_{n}^{\tilde{C}} \equiv\left\langle W_{n}|\overline{\mathscr{J} \tilde{C}}(0)| 0\right\rangle$, where $\overline{\mathscr{J} \tilde{C}}\left(t_{0}\right)$ stands for the source operator for $\tilde{C}$ which creates two-baryon states. The ellipses denote inelastic contributions coming from channels above $C$. The ground state of the NBS wave functions can be extracted for sufficiently large $t-t_{0}$, where contributions from all excited states can be neglected. In the practical calculation, however, we have two difficulties to obtain the ground state of NBS wave functions: (i) It is difficult to realize the ground state domination since $t-t_{0}$ cannot be taken large enough due to statistical noises of

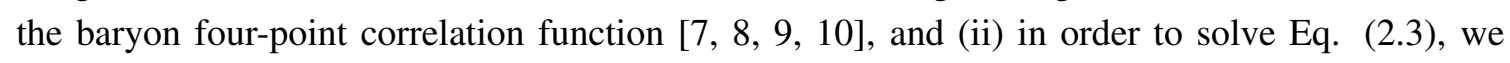
need not only the ground state of NBS wave functions but also the first excited state of NBS wave functions, which are difficult to isolate each other due to the same reasons as (i). The improved method to extract the potentials without using the ground state domination has been proposed in Ref. [U]] in the case of the single channel and extended to the coupled-channel case in Ref. [B], 团] as follows. Let us consider the normalized baryon four-point correlation function $R_{C}^{\tilde{C}}\left(\vec{r}, t-t_{0}\right) \equiv$ $G_{C}^{\tilde{C}}\left(\vec{r}, t-t_{0}\right) / \exp \left[-\left(m_{C_{1}}+m_{C_{2}}\right)\left(t-t_{0}\right)\right]$ which satisfies

$$
\left[\left(\frac{1+3 \delta_{C}^{2}}{8 \mu_{C}}\right) \frac{\partial^{2}}{\partial t^{2}}-\frac{\partial}{\partial t}-\left(H_{0}\right)_{C}\right] R_{C}^{\tilde{C}}\left(\vec{r}, t-t_{0}\right)=\sum_{C^{\prime}} \int d^{3} r^{\prime} \Delta_{C}^{C^{\prime}} U_{C}^{C^{\prime}}\left(\vec{r}, \vec{r}^{\prime}\right) R_{C^{\prime}}^{\tilde{C}}\left(\vec{r}^{\prime}, t-t_{0}\right),
$$

for a moderately large $t-t_{0}$ where inelastic contributions from channels other than $\Lambda_{c} N$ and $\Sigma_{c} N$ can be neglected. $\delta_{C}$ and $\Delta_{C}^{C^{\prime}}$ in Eq. (2.5) are defined by

$$
\begin{aligned}
\delta_{C} & =\left(m_{C_{1}}-m_{C_{2}}\right) /\left(m_{C_{1}}+m_{C_{2}}\right), \\
\Delta_{C}^{C^{\prime}} & =\sqrt{\left(Z_{C_{1}} Z_{C_{2}}\right) /\left(Z_{C_{1}^{\prime}} Z_{C_{2}^{\prime}}\right)} \exp \left[-\left(m_{C_{1}^{\prime}}+m_{C_{2}^{\prime}}-m_{C_{1}}-m_{C_{2}}\right)\left(t-t_{0}\right)\right] .
\end{aligned}
$$

We can then extract the potential matrix at the leading-order of the derivative expansion as

$$
\left(\begin{array}{cc}
\tilde{V}_{\Lambda_{c} N}^{\Lambda_{c} N}(\vec{r}) & \tilde{V}_{\Lambda_{c} N}^{\sum_{c} N}(\vec{r}) \\
\tilde{V}_{\Sigma_{c} N}^{\Lambda_{c} N}(\vec{r}) & \tilde{V}_{\Sigma_{c} N}^{\sum_{c} N}(\vec{r})
\end{array}\right)=\left(\begin{array}{cc}
\mathscr{K}_{\Lambda_{c} N}^{\Lambda_{c} N}\left(\vec{r}, t-t_{0}\right) & \mathscr{K}_{\Lambda_{c} N}^{\Sigma_{c} N}\left(\vec{r}, t-t_{0}\right) \\
\mathscr{K}_{\Sigma_{c} N}^{\Lambda_{c} N}\left(\vec{r}, t-t_{0}\right) & \mathscr{K}_{\Sigma_{c} N}^{\sum_{c} N}\left(\vec{r}, t-t_{0}\right)
\end{array}\right)\left(\begin{array}{cc}
R_{\Lambda_{c} N}^{\Lambda_{c} N}\left(\vec{r}, t-t_{0}\right) & R_{\Lambda_{c} N}^{\Sigma_{c} N}\left(\vec{r}, t-t_{0}\right) \\
R_{\Sigma_{c} N}^{\Lambda_{c} N}\left(\vec{r}, t-t_{0}\right) & R_{\Sigma_{c} N}^{\sum_{c} N}\left(\vec{r}, t-t_{0}\right)
\end{array}\right)^{-1}
$$

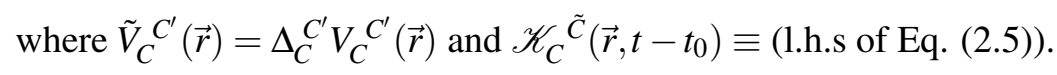




\section{Numerical setup}

For numerical simulations, we employ the $(2+1)$-flavor full QCD configurations generated by PACS-CS Collaboration [ [D2] with the renormalization group improved Iwasaki gluon action and a nonperturbatively $\mathscr{O}(a)$ improved Wilson-clover quark action at $\beta=6 / g^{2}=1.90$ on a $L^{3} \times T=$ $32^{3} \times 64$ lattice. The corresponding lattice spacing is $a=0.0907(13) \mathrm{fm}$ and the physical lattice size is $L a=2.902(42) \mathrm{fm}$. In order to see the quark mass dependence of the potentials, we employ three ensembles of gauge configurations corresponding to $m_{\pi}=700(2), 570(1)$ and 412(2) MeV. For the charm quark, we employ the relativistic heavy quark (RHQ) action [13]] to avoid the leading $\mathscr{O}\left(\left(m_{Q} a\right)^{n}\right)$ and the next-to-leading $\mathscr{O}\left(\left(m_{Q} a\right)^{n}\left(a \Lambda_{\mathrm{QCD}}\right)\right)$ discretization errors due to the charm quark mass $m_{Q}$. We use the RHQ parameters determined in Ref. [14]] so as to reproduce the experimental value of the mass and the relativistic dispersion relation for the charmonium in the spin-averaged $1 S$ state. We calculate quark propagators for a zero momentum wall source by imposing Coulomb gauge fixing at $t=t_{0}$ with the periodic boundary condition for the spatial directions, while the Dirichlet boundary condition is imposed on the temporal direction at the time-slice $t=32+t_{0}$. Four-point correlation functions are calculated using the unified contraction algorithm [ㄷ]]. In order to increase the statistics, we take an average over forward and backward propagations in time. Furthermore, we take 64 different time-slices for each configuration as the wall source location. For all analyses in this study, the jackknife method is employed to estimate statistical errors. The binsize of the jackknife samples is taken to 57,40 and 45 for the $m_{\pi} \simeq$ 700, 570 and $410 \mathrm{MeV}$ case, respectively. We confirm that change of binsize does not affect the errors for hadron masses as well as the errors for potentials and phase shifts. The number of gauge configurations for each ensemble and various hadron masses calculated in this work are

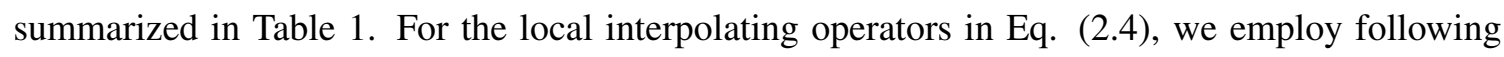

Table 1: The number of configurations and calculated hadron masses in unit of [MeV] on each ensemble.

\begin{tabular}{l|cccccc}
\hline \hline & \# of gauge configs. & $m_{\pi}$ & $m_{N}$ & $m_{\Lambda_{c}}$ & $m_{\Sigma_{c}}$ & $m_{\Sigma_{c}^{*}}$ \\
\hline Ensemble 1 & 399 & $702(2)$ & $1581(6)$ & $2685(3)$ & $2780(5)$ & $2866(5)$ \\
Ensemble 2 & 400 & $570(1)$ & $1399(9)$ & $2555(5)$ & $2674(7)$ & $2763(7)$ \\
Ensemble 3 & 450 & $412(2)$ & $1215(9)$ & $2434(6)$ & $2575(9)$ & $2661(10)$ \\
\hline \hline
\end{tabular}

form for a nucleon, $\Lambda_{c}$ and $\Sigma_{c}$ as

$$
B_{\alpha}(x)=\varepsilon_{i j k}\left[q_{i}^{T}(x) C \gamma_{5} q_{j}(x)\right] q_{k, \alpha}(x),
$$

where $x=(\vec{x}, t)$, and $i, j, k$ are color indices. $C$ is the charge conjugation matrix defined by $C=\gamma_{2} \gamma_{4}$, and $q=u, d, c$ stands for quark operators for up-, down- and charm-quarks, respectively. Flavor structures of a nucleon, $\Lambda_{c}$ and $\Sigma_{c}$ are given by

$$
\begin{aligned}
N & =\left(\begin{array}{c}
p \\
n
\end{array}\right)=\left(\begin{array}{c}
{[u d] u} \\
{[u d] d}
\end{array}\right), \quad \Sigma_{c}=\left(\begin{array}{c}
\Sigma_{c}^{++} \\
\Sigma_{c}^{+} \\
\Sigma_{c}^{0}
\end{array}\right)=\left(\begin{array}{c}
{[c u] u} \\
\frac{1}{\sqrt{2}}([c d] u+[c u] d) \\
{[c d] d}
\end{array}\right), \\
\Lambda_{c}^{+} & =\frac{1}{\sqrt{6}}([c d] u+[u c] d-2[d u] c),
\end{aligned}
$$




\section{Numerical results}
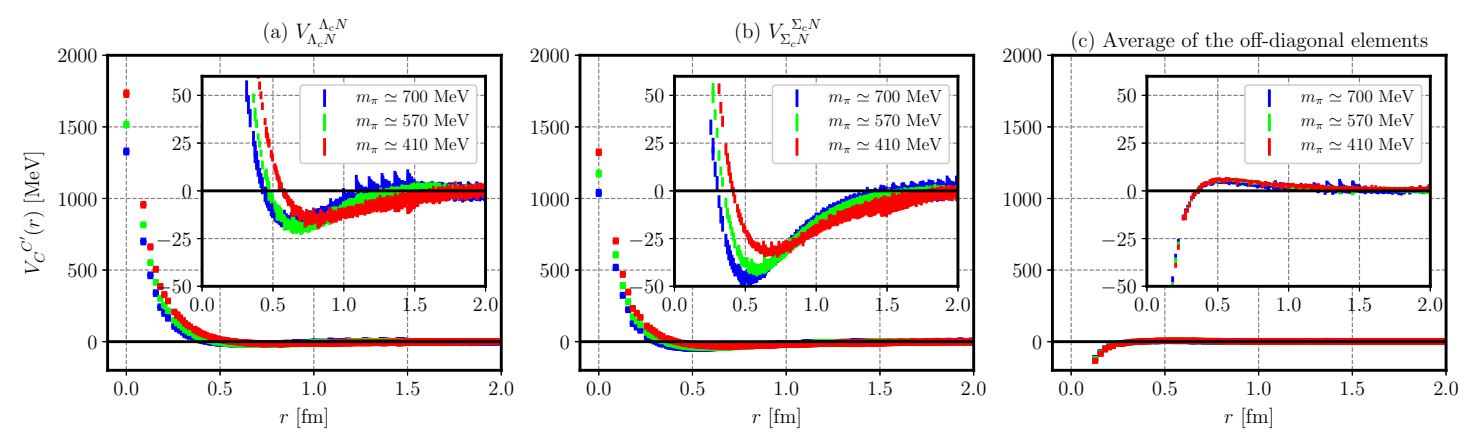

Figure 1: The leading order potentials in the derivative expansion for the S-wave $\Lambda_{c} N-\Sigma_{c} N$ coupledchannel system in $I\left(J^{P}\right)=\frac{1}{2}\left(1^{+}\right)$state. (a), (b) show that the diagonal elements, while the average of the off-diagonal elements is shown in (c). The potentials are calculated at $t-t_{0}=13,11,9$ for $m_{\pi} \simeq 700$ (Blue), 570 (Green) and $410 \mathrm{MeV}$ case (Red), respectively. The effect of relative the D-waves is included implicitly as the effective central potentials.

In Fig. 四, we show the S-wave $\Lambda_{c} N-\Sigma_{c} N$ coupled-channel potentials in the $I\left(J^{P}\right)=\frac{1}{2}\left(1^{+}\right)$ state calculated by using Eq. (2.8). The $A_{1}^{+}$projection of the cubic group is taken for the sink operator to obtain the relative $S$-wave in the NBS wave function. In the present results, relative D-waves are not explicitly considered but their effect is included implicitly as the effective central potentials. These potentials are calculated at $t-t_{0}=13$ (Ensemble 1: $m_{\pi} \simeq 700 \mathrm{MeV}$ ), $t-t_{0}=11$ (Ensemble 2: $m_{\pi} \simeq 570 \mathrm{MeV}$ ) and $t-t_{0}=9$ (Ensemble 3: $m_{\pi} \simeq 410 \mathrm{MeV}$ ). It is confirmed that these potentials are stable against the change of $t-t_{0}$ within the statistical errors. In Fig. 四, we observe a strong attraction in the $V_{\Sigma_{c} N}^{\sum_{c} N}$ potential, which might be a manifestation of the one-pion exchange, while the $V_{\Lambda_{c} N}^{\Lambda_{c} N}$ potential remains weak. Both diagonal potentials have strong pion-mass dependences. On the other hand, the off-diagonal potential is almost independent of pion masses.

We then calculate physical observables such as scattering phase shifts from the potentials. For this purpose, we fit the potential data with the three-range Gaussian $V(r)=\sum_{n=1}^{3} a_{n} \exp \left[-\left(r / b_{n}\right)^{2}\right]$. We solve the Schrödinger equation with the fitted potentials in the infinite volume and extract $S$ matrix from the asymptotic behavior of the wave functions. The definition of the phase shifts for the coupled channel system is given in Ref. [ए]] as

$$
\left(\begin{array}{cc}
S_{\Lambda_{c} N, \Lambda_{c} N} & S_{\Lambda_{c} N, \Sigma_{c} N} \\
S_{\Sigma_{c} N, \Lambda_{c} N} & S_{\Sigma_{c} N, \Sigma_{c} N}
\end{array}\right) \equiv\left(\begin{array}{cc}
\mathrm{e}^{i \bar{\delta}_{\Lambda_{c} N}} & 0 \\
0 & \mathrm{e}^{i \bar{\delta}_{\Sigma_{c} N}}
\end{array}\right)\left(\begin{array}{cc}
\cos 2 \bar{\theta} & i \sin 2 \bar{\theta} \\
i \sin 2 \bar{\theta} & \cos 2 \bar{\theta}
\end{array}\right)\left(\begin{array}{cc}
\mathrm{e}^{i \bar{\delta}_{\Lambda_{c} N}} & 0 \\
0 & \mathrm{e}^{i \bar{\delta}_{\Sigma_{c} N}}
\end{array}\right),
$$

where $\bar{\delta}$ is so-called the bar phase shift and $\bar{\theta}$ is the mixing angle. To retain the unitarity of the S-matrix, two off-diagonal elements of the potential matrix are replaced by their average, though the result obtained with the original potential matrix does not change within statistical errors. Fig. [ shows the phase shift of each channel and the inelasticity defined as $\left|S_{\Lambda_{c} N}^{\Lambda_{c} N}\right|=|\cos 2 \bar{\theta}|$. From Fig. ■, we find that there are no bound state for both $\Lambda_{c} N$ and $\Sigma_{c} N$ channels at all pion masses, even though the attraction of the $\Sigma_{c} N$ channel at low energies is stronger than that of the $\Lambda_{c} N$ channel. We also find the small inelasticity of the scattering, which indicates that the transition between the $\Lambda_{c} N$ and the $\Sigma_{c} N$ is weak and two channels are almost independent each other. This observation might be understood from the fact that the large mass splitting between the $\Lambda_{c} N$ and the $\Sigma_{c} N$. 

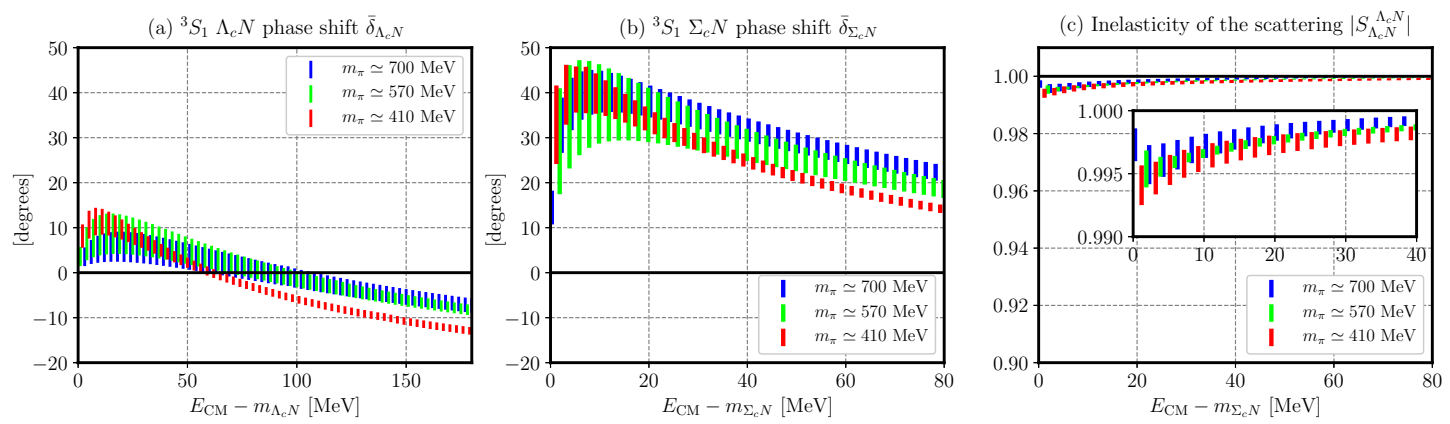

Figure 2: The phase shifts of the $\Lambda_{c} N$ channel (a), and $\Sigma_{c} N$ channel (b) in ${ }^{3} S_{1}$ state for each pion mass. The $\Lambda_{c} N\left(\Sigma_{c} N\right)$ phase shift are plotted against the energies from the $\Lambda_{c} N\left(\Sigma_{c} N\right)$ thereshold. The inelasticity of the scattering defined as $\left|S_{\Lambda_{c} N}^{\Lambda_{c} N}\right|=|\cos 2 \bar{\theta}|$ is plotted against the energies from the $\Sigma_{c} N$ thereshold (c).

\section{Summary and conclusion}

We have investigated the $S$-wave interaction in the $I\left(J^{P}\right)=\frac{1}{2}\left(1^{+}\right) \Lambda_{c} N-\Sigma_{c} N$ coupled-channel system on the basis of lattice QCD simulations. The potentials have been extracted by the coupledchannel HAL QCD method using the (2+1)-flavor full QCD configurations with the lattice volume of $(2.9 \mathrm{fm})^{3}$ and the pion mass, $m_{\pi} \simeq 700,570,410 \mathrm{MeV}$. Our numerical results show that both diagonal elements in the potential matrix are attractive in the long range and the strength of the attraction in $V_{\Sigma_{c} N}^{\Sigma_{c} N}$ is larger than that in $V_{\Lambda_{c} N}^{\Lambda_{c} N}$, probably due to the one-pion exchange. We have also observed off-diagonal elements are weak. The phase shifts and the inelasticity calculated from obtained potentials show that that the both channel does not have the two-body bound state at $m_{\pi} \geq 410 \mathrm{MeV}$, even though the net attraction of the $\Sigma_{c} N$ channel is stronger than that of the $\Lambda_{c} N$ channel. The inelasticity of the scattering also indicates a small coupling between the two.

\section{Acknowledgments}

We thank the PACS-CS Collaboration for providing us their $2+1$ flavor gauge configurations [ए2]. Numerical computations of this work have been carried out by the KEK supercomputer system (BG/Q), [Project number : 15/16-12].

\section{References}

[1] S. Aoki, T. Hatsuda and N. Ishii, Prog. Theor. Phys. 23 (2010) 89.

[2] S. Aoki et al. [HAL QCD Collaboration], Prog. Theor. Exp. Phys. 2012 (2012) 01A105.

[3] S. Aoki, B. Charron, T. Doi, T. Hatsuda, T. Inoue and N. Ishii, Phys. Rev. D 87 (2013) 034512.

[4] K. Sasaki et al. [HAL QCD Collaboration], Prog. Theor. Exp. Phys. 2015 (2015) 113 B01.

[5] Y. Ikeda et al. [HAL QCD Collaboration], Phys. Rev. Lett. 117, 242001 (2016).

[6] T. Miyamoto et al. [HAL QCD Collaboration], arXiv:1710.05545 [hep-lat].

[7] T. Iritani et al. [HAL QCD Collaboration], JHEP 1610, 101 (2016).

[8] S. Aoki [HAL QCD Collaboration], PoS LATTICE 2016, 109 (2016) [arXiv:1610.09763 [hep-lat]].

[9] T. Iritani [HAL QCD Collaboration], PoS LATTICE 2016, 107 (2016) [arXiv:1610.09779 [hep-lat]].

[10] T. Iritani et al. [HAL QCD Collaboration], Phys. Rev. D96 (2017) no.3, 034521.

[11] N. Ishii et al. [HAL QCD Collaboration], Phys. Lett. B712 (2012) 437.

[12] PACS-CS Collaboration: S. Aoki, et al., Phys. Rev. D79 (2009) 034503.

[13] S. Aoki, Y. Kuramashi and S. Tominaga, Prog. Theor. Phys 109 (2003) 383.

[14] Y. Namekawa, et al. (PACS-CS Collaboration), Phys. Rev. D87, 094512.

[15] T. Doi and M. G. Endres, Comput. Phys. Commun. 184, 117 (2013).

[16] H. Stapp, T. J. Ypsilantis and N. Metropolis, Phys. Rev. 105 (1957) 302. 